\title{
A NUMERICAL TECHNIQUE FOR SOLVING CARRIER-MEDIATED TRANSPORT PROBLEMS
}

\author{
RAVI JAIN and JEROME S. SCHULTZ*
}

Department of Chemical Engineering, The University of Michigan, Ann Arbor MI 48109 (U.S.A.)

(Received September 25, 1981; accepted in revised form November 5, 1981)

\section{Summary}

A numerical technique and a computer program based on the method of orthogonal collocation on finite elements for solving steady state carrier-mediated transport problems is presented. The method is especially suitable in solving boundary layer type of problems as arise in carrier-mediated transport. The method uses an efficient $L U$ decomposition of a blockdiagonal matrix. A residual criterion is used for the placement of elements which can be easily implemented using an interactive feature in the computer program. Numerical results for three different systems; a system with simple kinetics and equal diffusivities for carrier and its complex, a system with simple kinetics and unequal diffusivities and a system with complex kinetics and equal diffusivities, indicate that the method is very versatile and has very good convergence properties. The method can be used very easily for all these systems by making minor modifications to the computer program.

\section{Introduction}

The phenomenon of carrier-mediated transport, which involves diffusional transport coupled with chemical reactions, has been known for a long time. The major impetus for the work in this area was provided by the Scholander's [1] study of oxygen transport in hemoglobin solutions. It has since become an important area of study for engineers due to its applicability to various biological and non-biological systems [2-7]. The analysis of carrier-mediated transport involves the solution of a system of second order non-linear differential equations of the boundary value type and has enjoyed considerable attention since no exact analytical solutions exist. Two approaches have been used for the solution; semi-analytical and numerical. The semi-analytical methods involve the techniques based on matched asymptotic expansions [8-11], singular perturbation method [30], regular perturbation method $[31,32]$ and techniques involving other approximations to the differential equations [12-14]. The numerical methods involve the quasi-linearization technique $[15,16]$, a finite difference technique with non-uniform mesh

\footnotetext{
*To whom all correspondence about the paper should be addressed.
} 
points [32] and a technique based on the Galerkin method [17]. The semianalytical solutions are elegant and easy to apply provided their validity has been established, usually by some numerical solution. Their range of applicability is also often not known beforehand. The numerical methods, on the other hand, can be used as general purpose tools for solving these problems.

In this paper, we describe a technique based on orthogonal collocation on finite elements introduced by Carey and Finlayson [18] and a computer program based on the technique. The method is particularly suitable for solving the boundary layer type of problems which usually arise in the analysis of carrier-mediated transport systems. The method uses a very efficient storage and computational scheme as is discussed later. The method can also be applied to a diverse class of carrier-mediated transport problems, some of which are the facilitated transport in composite membranes [19-21], facilitated transport with competing permeants $[22,19]$, facilitated transport involving reversible as well as irreversible reaction [23] and the classical carrier-mediated transport problem [11]. The use of the technique is demonstrated in this paper using three different carrier-mediated transport systems. Comparisons with other numerical techniques are also made. Applications of the technique to other situations will be discussed in a forthcoming paper.

\section{Mathematical description of the carrier-mediated transport problem}

We start by considering the situation in which a reversible chemical reaction takes place between a permeant, A, and a carrier, B, to form a carrierpermeate complex, AB. The carrier and its complex are constrained to stay within a membrane of thickness, $L$. The diffusion reaction equations describing the system can be written as:

$D_{i} \frac{\mathrm{d}^{2} C_{i}}{\mathrm{~d} x^{2}}=r_{i} \quad(i=\mathrm{A}, \mathrm{B}, \mathrm{AB})$

where $C_{i}, D_{i}$ and $r_{i}$ are the concentrations, diffusivities and reaction rates, respectively, and

$r_{\mathrm{A}}=r_{\mathrm{B}}=-r_{\mathrm{AB}}=k_{1} C_{\mathrm{A}} C_{\mathrm{B}}-k_{2} C_{\mathrm{AB}}$

$k_{1}$ and $k_{2}$ are the forward and the reverse rate constants for the reaction

$\mathrm{A}+\mathrm{B} \rightleftharpoons \mathrm{AB}$

The boundary conditions are

$C_{\mathrm{A}}=C_{\mathrm{A}}{ }^{0} \quad$ at $x=0$

$C_{\mathrm{A}}=C_{\mathrm{A}}{ }^{L} \quad$ at $x=L$

and 
$\frac{\mathrm{d} C_{\mathrm{B}}}{\mathrm{d} x}=\frac{\mathrm{d} C_{\mathrm{AB}}}{\mathrm{d} x}=0 \quad$ at $x=0 \& L$

All of these conditions are not independent and an extra condition is obtained by making a balance on the total amount of carrier in free and the associated forms. The balance equation can be written as

$$
\int_{0}^{L}\left(C_{\mathrm{B}}+C_{\mathrm{AB}}\right) \mathrm{d} x=C_{\mathrm{T}} L
$$

where $C_{\mathrm{T}}$ is the average total concentration of $\mathrm{B}$ in all its forms. Writing eqn. (1) for $i=\mathrm{B}$ and $\mathrm{AB}$, adding the resulting equations, integrating across the membrane and applying the boundary conditions in eqn. (3) gives:

$D_{\mathrm{B}} \frac{\mathrm{d} C_{\mathrm{B}}}{\mathrm{d} x}+D_{\mathrm{AB}} \frac{\mathrm{d} C_{\mathrm{AB}}}{\mathrm{d} x}=0$.

Further integration gives,

$D_{\mathrm{B}} C_{\mathrm{B}}+D_{\mathrm{AB}} C_{\mathrm{AB}}=C^{*}$

where $C^{*}$ is an integration constant. Equation (6) can be rewritten as:

$D_{\mathrm{AB}}\left(C_{\mathrm{AB}}+C_{\mathrm{B}}\right)+\left(D_{\mathrm{B}}-D_{\mathrm{AB}}\right) C_{\mathrm{B}}=C^{*}$

Substitution of the value of $\left(C_{\mathrm{AB}}+C_{\mathrm{B}}\right)$ from eqn. (7) in eqn. (4) and rearrangement gives the defining relation for $C^{*}$ :

$C^{*}=D_{\mathrm{AB}} C_{\mathrm{T}}+\frac{\left(D_{\mathrm{B}}-D_{\mathrm{AB}}\right)}{L} \int_{0}^{L} C_{\mathrm{B}} \mathrm{d} x$.

Eqn. (7) is the balance equation for the total amount of carrier for general case of non-equal diffusivities. In most of the cases treated in the literature the carrier and its complex are of about the same size and the equal diffusivity assumption can be used with little error, i.e. $D_{B}=D_{A B}$, and application of eqn. (8) to eqn. (7) for this case gives:

$C_{\mathrm{B}}+C_{\mathrm{AB}}=C_{\mathrm{T}}$.

The numerical procedure developed in this paper is illustrated for the case of equal, as well as unequal diffusivities. For either case we have an algebraic relation between $C_{\mathrm{B}}$ and $C_{\mathrm{AB}}$, and eqn. (1) need be solved only for the components $A$ and $B$, which for the case of equal diffusivities reduces to:

$D_{\mathrm{A}} \frac{\mathrm{d}^{2} C_{\mathrm{A}}}{\mathrm{d} x^{2}}=k_{1} C_{\mathrm{A}} C_{\mathrm{B}}-k_{2}\left(C_{\mathrm{T}}-C_{\mathrm{B}}\right)$

and 
$D_{\mathrm{B}} \frac{\mathrm{d}^{2} C_{\mathrm{B}}}{\mathrm{d} x^{2}}=k_{1} C_{\mathrm{A}} C_{\mathrm{B}}-k_{2}\left(C_{\mathrm{T}}-C_{\mathrm{B}}\right)$

De-dimensionalization of eqns. (10) and (11) gives:

$\frac{\mathrm{d}^{2} \bar{C}_{\mathrm{A}}}{\mathrm{d} \bar{x}^{2}}=P\left(Q \bar{C}_{\mathrm{A}} \bar{C}_{\mathrm{B}}-T+\bar{C}_{\mathrm{B}}\right)$

and

$\frac{\mathrm{d}^{2} \bar{C}_{\mathrm{B}}}{\mathrm{d} \bar{x}^{2}}=S\left(Q \bar{C}_{\mathrm{A}} \bar{C}_{\mathrm{B}}-T+\bar{C}_{\mathrm{B}}\right)$

where

$P=k_{2} L^{2} / D_{\mathrm{A}}, \quad S=k_{2} L^{2} / D_{\mathrm{B}}, \quad Q=k_{1} C_{\mathrm{A}}^{0} / k_{2}$ and $T=C_{\mathrm{T}} / C_{\mathrm{A}}^{0}$.

$P$ and $S$ are the two Damköhler numbers for the system. The relations between the de-dimensionalized and the original variables are:

$\bar{C}_{\mathrm{A}}=C_{\mathrm{A}} / C_{\mathrm{A}}^{0}, \bar{C}_{\mathrm{B}}=C_{\mathrm{B}} / C_{\mathrm{A}}^{0}$ and $\bar{x}=x / L$.

The boundary conditions reduce to

$\bar{C}_{\mathrm{A}}=1 \quad$ at $\bar{x}=0$

$\bar{C}_{\mathrm{A}}=C_{\mathrm{A}}^{L} / C_{\mathrm{A}}^{0}$ at $\bar{x}=1$

and

$\frac{\mathrm{d} \bar{C}_{\mathrm{B}}}{\mathrm{d} \bar{x}}=0 \quad$ at $\bar{x}=0,1$

The physical quantities of interest after solving these equations are the total flux of the permeating species, A, and the facilitation factor, $F$, defined as the ratio of the facilitated flux and the flux due to the simple physical diffusion alone. These can be obtained by subtracting eqns. (10) and (11), integrating and applying the boundary conditions in eqn. (3) and then integrating once more as illustrated by Smith et al. [11]. The procedure gives following relation for the total flux, $N_{\mathrm{A}}$, of the permeant:

$N_{\mathrm{A}}=\frac{D_{\mathrm{A}} C_{\mathrm{A}}^{0}}{L}\left[\left(1-\bar{C}_{\mathrm{A}}^{1} / \bar{C}_{\mathrm{A}}^{0}\right)+\frac{D_{\mathrm{B}}}{D_{\mathrm{A}}}\left(\bar{C}_{\mathrm{B}}^{1}-\bar{C}_{\mathrm{B}}^{0}\right)\right]$

where

$\bar{C}_{\mathrm{A}}^{1} / \bar{C}_{\mathrm{A}}^{0}=C_{\mathrm{A}}^{L} / C_{\mathrm{A}}^{0}$.

The facilitation factor, $F$, is given as 
$F=\frac{D_{\mathrm{B}}}{D_{\mathrm{A}}} \frac{\left(\bar{C}_{\mathrm{B}}^{1}-\bar{C}_{\mathrm{B}}^{0}\right)}{\left(1-C_{\mathrm{A}}^{\mathrm{L}} / C_{\mathrm{A}}^{0}\right)}$

Thus both the flux and the facilitation factor can be calculated once the boundary concentrations of free carrier are determined, i.e. $\bar{C}_{B}^{1}$ and $\bar{C}_{B}^{0}$.

\section{The method of orthogonal collocation on finite elements}

In the method of orthogonal collocation on finite elements, the domain over which the solution is to be obtained is divided in a number of elements (which for the present case involves dividing the line $0 \leqslant x \leqslant 1$ in segments of different lengths). The method involves the application of the method of global orthogonal collocation for each element. The solutions for different elements are joined together by requiring the continuity across the elements. A brief discussion of the methods of orthogonal collocation $[24,25,33]$ and orthogonal collocation on finite elements [18] is given next.

Consider a second order ordinary differential equation:

$\frac{d^{2} y}{d x^{2}}=f(x, y)$.

The boundary conditions for the differential equation are assumed to be known. The method of orthogonal collocation consists in representing the solution to the differential equation by a linear combination of orthogonal polynomials in the interior of the interval. A typical solution is,

$y(x)=a+b x+x(1-x) \sum_{i=1}^{n} a_{i} P_{i-1}(x)$

where $P_{i-1}(x)$ 's are the orthogonal polynomials of $(i-1)$ th order defined over the interval of interest and the first two terms are added to account for the boundary conditions. Shifted Legendre polynomials defined over the interval $0 \leqslant x \leqslant 1$ were chosen in this work. The $N$ th degree polynomial has $N$ roots in the interval. Equation (18) contains $N+2$ unknown constants and can alternatively be written as:

$y(x)=\sum_{i=1}^{N+2} d_{i} x^{i-1}$

where $d_{i}$ 's are $N+2$ unknown constants. The roots of the polynomial $P_{N}(x)$ in the interior of the domain are taken as the collocation points. The reason for doing this is explained after setting up the equations for the system. If we, for a moment, assumathat the solutions $y\left(x_{i}\right)$ 's at the collocation points $x_{j}$ 's are known, eqn. (19) can be rewritten as:

$y\left(x_{j}\right)=\sum_{i=1}^{N+2} d_{i} x_{j}^{l-1}$. 
This equation can be used to derive the expressions for the first and second derivatives at the collocation points in terms of the solutions at the collocation points and the roots of the orthogonal polynomials. Successive differentiations of eqn. (19) and evaluations at $x=x_{j}$ give:

$$
\begin{aligned}
& \frac{\mathrm{d} y}{\mathrm{~d} x}\left(x_{j}\right)=\sum_{i=1}^{N+2} d_{i}(i-1) x_{j}^{i-2} \\
& \frac{\mathrm{d}^{2} y}{\mathrm{~d} x^{2}}\left(x_{j}\right)=\sum_{i=1}^{N+2} d_{i}(i-1)(i-2) x_{j}^{i-3}
\end{aligned}
$$

Equations (20) and (21) can be written in matrix form as

$\bar{y}=\overline{\bar{D}} \cdot \bar{d}, \quad \frac{\overline{\mathrm{d} y}}{\mathrm{~d} x}=\overline{\bar{E}} \cdot \bar{d}$ and $\frac{\overline{\mathrm{d}^{2} y}}{\mathrm{~d} x^{2}}=\bar{F} \cdot \bar{d}$

where

$D_{j i}=x_{j}{ }^{i-1}, \quad E_{j i}=(i-1) x_{j}^{i-2}$ and $\quad F_{j t}=(i-1)(i-2) x_{j}^{i-3}$

From eqn. (22) we have

$\bar{d}=\bar{D}^{-1} \bar{y}$

and hence

$\frac{\overline{\mathrm{d} y}}{\mathrm{~d} x}=\overline{\bar{E}} \cdot \bar{D}^{-1} \cdot \bar{y}=\bar{A} \cdot \bar{y}$

$\frac{\overline{\mathrm{d}^{2} y}}{\mathrm{~d} x^{2}}=\bar{F} \cdot \bar{D}^{-1} \bar{y}=\bar{B} \cdot \bar{y}$

The square matrices $A$ and $B$ are dependent solely on the roots of the orthogonal polynomials and hence can be calculated once and for all as soon as the orthogonal polynomials are selected. This is a direct consequence of choosing the roots of orthogonal polynomials as the collocation points. An arbitrary choice of collocation points would require re-evaluation of $A$ and $B$ matrices for each problem. It is also seen in (ref. 25) that with this procedure, the solution converges very rapidly as the order of polynomial, $N$, is increased. This is another reason for choosing the roots as the collocation points.

If the integral of the solution vector $\bar{y}$ is desired, it can be evaluated using the quadrature formula,

$$
\int_{0}^{1} y(x) \mathrm{d} x=\sum_{i=1}^{N+2} W_{j} y\left(x_{j}\right)
$$


Equation (26) with $y_{i}=x^{i-1}$ gives

$\bar{W} \cdot \bar{D}=\bar{G}$

where

$G_{i}=1 / i$

so

$\bar{W}=\bar{G} \cdot \overline{\bar{D}}^{-1}$

The matrices $A, B$, and the vector $W$ are given by Finlayson $[25,33]$. The derivatives can now be substituted in the differential eqn. (17) to form the algebraic equations of the type

$$
\sum_{i=1}^{N+2} B_{j i} y_{i}-f\left(x_{j}, y_{j}\right)=0 \quad j=2, N+1
$$

These equations are known as the residual equations. Two more equations can be obtained if the boundary conditions are specified. If, for example, $y=a$ at $x=0$ and $\mathrm{d} y / \mathrm{d} x=b$ at $x=1$, we have two additional equations.

$y_{1}=a$

and

$\sum_{i=1}^{N+2} A_{N+2, i} y_{i}=b$

Equations (28) and (29) represent a set of $N+2$ equations in $N+2$ unknowns and can be solved to obtain the values of $y_{i}$. The solution thus obtained satisfies the differential equation exactly at the collocation points. The difference is usually small at other points. As the order of the polynomial, $N$, is increased, the differential equation is satisfied at more and more points and the error in the solution decreases consequently. Application of this method to several problems $[25,33]$ has indicated that the solutions obtained even with small $N$ are fairly accurate. However, in problems involving boundary layer type of solutions where the solution changes very rapidly over a small interval, as is the case with the most carrier-mediated transport problems, the usual orthogonal collocation procedures just outlined do not work well. This is due to the fact that even with very large $N$, very few, if any, collocation points are located in the boundary layer region where the solution is the most important. However if the domain is divided into number of elements, enough points can be located in the boundary layer region by locating the elements there and fairly accurate solutions can be found. The idea is illustrated by applying it to the carrier-mediated transport problem discussed in the previous section. Following Carey and Finlayson [18], the domain from $x=0$ to 1 is divided into $M$ elements of length $\Delta X_{l}, l=1, M$. A new variable, $u$, is defined 
in each element, $u^{l}=\left(x-x_{l}\right) / \Delta X_{l}$ and $\Delta X_{l}=x_{l+1}-x_{l}$. The variable $u$ goes from 0 to 1 in each element and the collocation points are the roots of $P_{N}(u)=$ 0 , where $P_{N}(u)$ are the shifted Legendre polynomials defined over the interval 0 to 1 . The bars on the concentrations will be omitted from now on for convenience. It should, however, be kept in mind that these variables are still dimensionless. The residual equations for the finite element formulation can be written as:

$\frac{1}{\Delta X_{l}^{2}} \sum_{i=1}^{N+2} B_{j i} C_{A_{i}^{l}}^{\prime}=P\left(Q C_{A_{j}^{l}}^{l} C_{B_{j}^{\prime}}^{l}-T+C_{\mathrm{B}_{j}}^{l}\right) \quad l=1, \ldots, M ; j=2, \ldots, N+1$

A similar equation holds for $C_{\mathrm{B}}$. The boundary conditions reduce to:

$C_{\mathrm{A}_{1}}^{1}=1$

$C_{\mathrm{A}_{N+2}^{M}}^{M}=C_{\mathrm{A}}^{L} / C_{\mathrm{A}}^{0}$

$\frac{1}{\Delta X_{1}} \sum_{i=1}^{N+2} A_{1 i} C_{B_{i}}^{1}=0$ and $\frac{1}{\Delta X_{M}} \sum_{i=1}^{N+2} A_{N+2, i} C_{B_{i}}^{M}=0$

The continuity of the dependent variables and the first derivatives across the elements leads to:

$C_{\mathrm{A}_{N+2}}^{l}=C_{\mathrm{A}_{1}^{l+1}}^{l+1}$

$\frac{1}{\Delta X_{l}} \sum_{i=1}^{N+2} A_{N+2, l} C_{\mathrm{A}_{i}}^{l}-\frac{1}{\Delta X_{l+1}} \sum_{i=1}^{N+2} A_{1, l} C_{\mathrm{A}_{i}^{l+1}}^{l+}=0 \quad l=1, \ldots, M-1$

A similar equation can be written for $C_{\mathrm{B}}$. Equations (30)-(32) represent a blockdiagonal system of equations as is discussed in detail by Carey and Fin. layson [18]. The solution to these equations represents the solution to the original problem for $M$ number of elements. As one places additional elements in appropriate places according to the criterion discussed later, the true solution is approached fairly rapidly. The method of solution is discussed next.

The method of solution

An orthogonal collocation procedure with 3 interior collocation points in each element (solution being represented by a fourth order polynomial) was used in this work. Before attempting the solution of the system of non-linear equations just discussed, the various unknowns $C_{\mathbf{A}_{i}^{l}}^{l}$ and $C_{\mathbf{B}_{i}^{l}}^{l}$ must be arranged in a single solution vector. This can be done as illustrated in Fig. 1 for 2 equally spaced elements. The numerical values of the actual collocation points are also shown in the figure. We remark that the collocation points are not equally spaced in each element. The $y_{i}$ 's in Fig. 1 represent the solution vector 


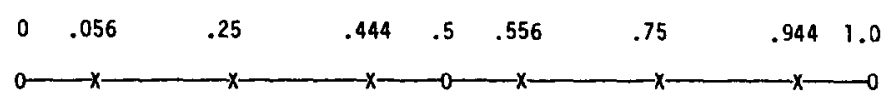

$\begin{array}{lllllllll}c_{A_{1}}^{\prime} & c_{A_{2}}^{1} & c_{A_{3}}^{1} & c_{A_{4}}^{1} & c_{A_{5}}^{1} & & & & \\ & & & C_{A_{1}}^{2} & C_{A_{2}}^{2} & c_{A_{3}}^{2} & c_{A_{4}}^{2} & c_{A_{5}}^{2}\end{array}$

$\begin{array}{lllll}C_{B_{1}}^{\prime} & C_{B_{2}}^{\prime} & C_{B_{3}}^{1} & C_{B_{4}}^{\prime} & C_{B_{5}}^{\prime}\end{array}$

$\begin{array}{lllll}c_{B_{1}}^{2} & c_{B_{2}}^{2} & c_{B_{3}}^{2} & c_{B_{4}}^{2} & c_{B_{5}}^{2}\end{array}$

$\begin{array}{llllllllll}C_{A}: & y_{1} & y_{3} & y_{5} & y_{7} & y_{9} & y_{11} & y_{13} & y_{15} & y_{17} \\ C_{B}: & y_{2} & y_{4} & y_{6} & y_{8} & y_{10} & y_{12} & y_{14} & y_{16} & y_{18}\end{array}$

Fig. 1. Location of collocation and element end points and arrangement of solution vector. $(0$, the element end points and the boundary points; $X$, the collocation points).

for the entire system. This kind of staggered arrangement is very helpful in writing the computer program. The approach can be easily generalized for more than two equations.

The Newton-Raphson method of Carnahan et al. [26] was used for the solution of the system of non-linear equations as large gradients in concentrations near the boundaries were anticipated. If the equation set is written as:

$\bar{F}(\bar{y})=0$

The Newton-Raphson procedure gives

$\overline{\bar{J}}\left(\bar{y}^{k+1}-\bar{y}^{k}\right)=-\bar{F}\left(\bar{y}^{k}\right)$

where $J$ is the Jacobian matrix for the system (also blockdiagonal) and $\bar{y}^{k}$, $\bar{y}^{k+1}$ are the values of $\bar{y}$ after $k$ th and $(k+1)$ th iteration respectively. Equation (34) represents a blockdiagonal system of linear equations. The major advantage of the present finite element method results from an efficient solution to this system of equations. For this purpose, an efficient general purpose routine to perform LU (lower-upper) decomposition with row equilibration and partial pivoting for blockdiagonal matrices was written. The method consists in applying the LU decomposition procedures [27] for dense matrices to each block in turn. Precautions are taken to ensure that no fills are generated outside the individual blocks during the elimination procedure. The diagonal blocks of the $J$ matrix and its LU decomposition are overstored over each other in a three dimensional array. The storage requirements and the computational efforts remain proportional to the number of elements with this procedure and the higher order solutions (solutions with large number of elements) do not become very expensive. 
Once the solution to eqn. (34) is obtained, the new solution can be calculated. The procedure is continued until the difference between the successive iterates $\left(\bar{y}^{k+1}-\bar{y}^{k}\right)$ becomes fairly small. This then represents the solution to the problem for $M$ number of elements at the collocation and the continuation points. To obtain solution at other points one can interpolate for each element using the following equation, similar equation being valid for $C_{\mathrm{B}}$.

$C_{\mathrm{A}}^{l}(x)=\sum_{i=1}^{N+2} d_{i}^{l} x^{i-1}$

Knowing the solution at the collocation and continuation points one can calculate the coefficients $d_{i}^{l}$ and hence the solution at other points. The solution can be improved upon by calculating the mean squared residual for each element as discussed by Carey and Finlayson [18]. However, as pointed out by them, the maximum residual is likely to occur at the continuation points. So the evaluation of residual at the continuation points suffices in most cases. This can be done by evaluating eqn. (30) for $j=1$ or $N+2$. Once the residuals at element end points have been calculated, additional elements are placed where the residuals are large. The collocation points for the new solution can then be calculated and the new starting guesses can be found using the interpolation formulae such as eqn. (35) calculated for the previous solution. The procedure is continued until the solution converges.

As mentioned earlier, the method of orthogonal collocation on finite elements with 3 interior collocation points in each element was used. A method with more interior points in each element would probably lead to a faster convergence requiring lesser number of elements for the final convergent solution but the consequent increase in the matrix size for individual blocks ( $10 \times 10$ for $N=3$ to $14 \times 14$ for $N=5$ ) increases the computational costs offsetting the advantage. The lower order method also has a lower round-off error, making it more desirable to use. A brief discussion of the computer program used in the solution is given next.

\section{The computer program}

The computer program, CARMED*, for the solution of the carrier-mediated transport problem consists of a set of thirteen routines, including the MAIN program. The arrangement of various subroutines is shown in Fig. 2 below. A downward sloping line indicates that a lower order subroutine is called by a higher order one. The functions of various subroutines are summarized below.

*A copy of the computer program and the users' manual is available from the authors. 


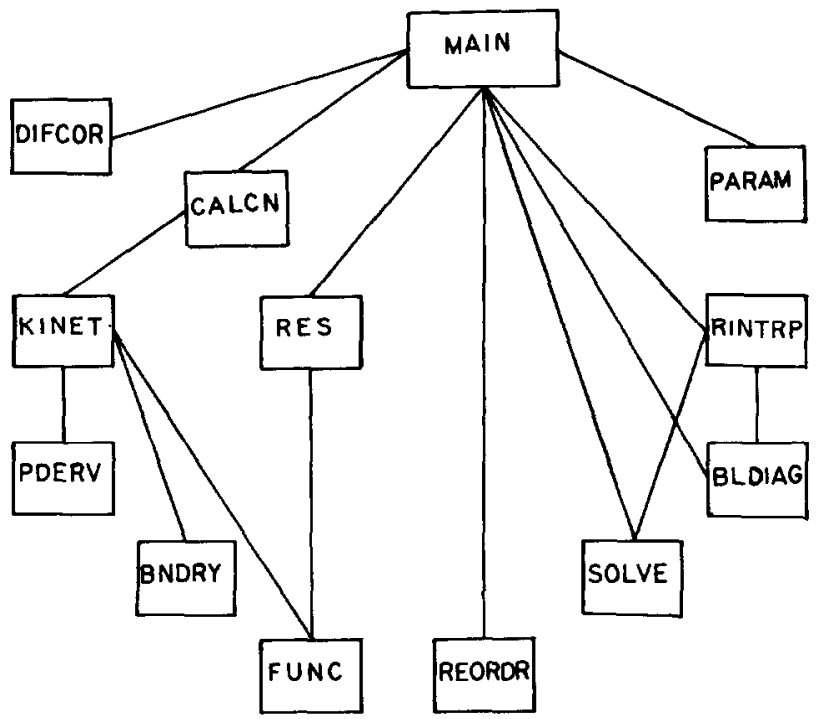

Fig. 2. Arrangement of various routines in the computer program, CARMED.

\section{MAIN}

The main program reads the input data, calls routine PARAM to calculate the parameters of the differential equations such as $P, Q$ etc., calls routine CALCN to set up the jacobian matrix, and the vector of the function values. It then calls routines BLDIAG and SOLVE to calculate the change in the solution vector, checks for the convergence and if the convergence is obtained calls routine RES to calculate the residuals at the continuation points and routine RINTRP to calculate the interpolating polynomials. Routine DIFCOR is called next if the diffusivities of carrier and its complex are not same. The MAIN program then reads the location of the additional elements interactively and calls routine REORDR to calculate the new starting solution. The whole process is then repeated until an exit route is taken.

\section{CALCN}

This routine sets up the jacobian matrix and the vector of function values for the blockdiagonal system of equations. Repeated calls to routine KINET are made to calculate the elements of the jacobian matrix and the function values corresponding to the boundary conditions and the reaction rate terms.

\section{BLDIAG}

This routine performs LU decomposition of a blockdiagonal matrix. Row equilibration and partial pivoting are performed in each block to reduce the roundoff errors.

\section{SOLVE}

This routine solves the blockdiagonal system of equations using the $L$ and $U$ 
matrices calculated by BLDIAG. Backward and forward substitution algorithms are used for this purpose.

RES

This routine calculates the residuals at the continuation points using the solution vector calculated by SOLVE. Routine FUNC is called to calculate the reaction rates at the collocation points.

RINTRP

This routine sets up the equations to calculate the interpolating polynomials for all the elements and then calls BLDIAG and SOLVE (with $N_{i}^{i}=1$ ) to solve them.

\section{REORDR}

This routine receives the location and the number of additional elements from MAIN program and using the interpolating polynomials from RINTRP, calculates the starting guesses in the Newton-Raphson procedure for the new set of equations.

\section{KINET}

This routine calculates the elements of the jacobian matrix, by calling routine PDERV, and the function values for the boundary conditions and the reaction rate terms, by calling routines FUNC \& BNDRY.

\section{PARAM}

This routine calculates the dimensionless parameters of the differential equations using the physical constants supplied by the MAIN program.

\section{FUNC}

This routine calculates the values of the kinetic expressions (right hand side of the differential equations) for various components.

\section{PDERV}

This routine calculates the partial derivatives of the kinetic expressions.

\section{$B N D R Y$}

This routine is used to define the boundary concentrations of the permeant.

\section{DIFCOR}

This routine is used to make corrections when the diffusivities of the carrier and its complex are not same.

Routines PARAM, PDERV, BNDRY \& DIFCOR are the ones that need to be changed for problems with different kinetic schemes. The procedure to construct these routines is illustrated in the Appendix. The users' manual 
contains these routines and complete set of data for three examples discussed in this paper.

\section{Results of the numerical calculations}

The computer program was tested for three different physical systems. The numerical results for two of these systems are available in the literature. The calculations were done on Amdahl $470 \mathrm{~V} / 8$ computer at the University of Michigan. The machine has a 16 digit accuracy in double precision. The major features of the present computational procedure are illustrated using the case of $\mathrm{CO}$ diffusion through hemoglobin solution. The other two systems $\left(\mathrm{CO}_{2}\right.$ diffusion through bicarbonate solution and NO diffusion through ferrous chloride solution) are used to illustrate the additional features as well as the application to situations not covered for CO facilitation. The results of the numerical calculations are discussed next.

\section{Carbon monoxide diffusion through membranes containing hemoglobin solution}

This system was experimentally studied by Mochizuki and Forster [22] and Wittenberg [28]. Numerical [15,32] as well as approximate analytical $[11,29,30]$ solutions are available. We first solved the equations describing the system using physical constants of Kutchai et al. [15]. Table 1 compares our results with the results of Kutchai et al. [15]. It can be clearly seen from this table that very large membrane thicknesses of about $2 \mathrm{~cm}$ are needed for $\mathrm{CO}-\mathrm{Hb}$ system to reach facilitations close to the asymptotic limit of

\section{TABLE 1}

The dependence of $\mathrm{CO}$ facilitation* on layer thickness $\left(P_{\mathrm{CO}}(0)=5 \mathrm{mmHg}\right.$ and $\left.P_{\mathrm{CO}}(L)=0\right)$

\begin{tabular}{rrllll}
\hline \multicolumn{1}{l}{$\begin{array}{l}L \\
(\mu \mathrm{m})\end{array}$} & $M$ & $\begin{array}{l}C_{\mathrm{B}}(0) / C_{\mathrm{A}}{ }^{0} \\
\left(\times 10^{-2}\right)\end{array}$ & $\begin{array}{l}C_{\mathrm{B}}(L) / C_{\mathrm{A}}{ }^{0} \\
\left(\times 10^{-2}\right)\end{array}$ & $F^{\dagger}$ & $F^{\S}$ \\
\hline 10 & 7 & 0.579 & 0.876 & 0.743 & 0.750 \\
50 & 10 & 0.448 & 1.96 & 3.77 & 3.75 \\
100 & 11 & 0.415 & 2.87 & 6.15 & 6.13 \\
150 & 13 & 0.400 & 3.56 & 7.89 & 7.84 \\
300 & 15 & 0.379 & 5.00 & 11.55 & 11.44 \\
500 & 15 & 0.368 & 6.23 & 14.65 & 14.44 \\
1000 & 19 & 0.357 & 7.96 & 19.01 & - \\
2000 & 19 & 0.349 & 9.53 & 22.95 & - \\
5000 & 23 & 0.344 & 11.02 & 26.70 & - \\
10000 & 26 & 0.341 & 11.69 & 28.38 & - \\
20000 & 26 & 0.340 & 12.08 & 29.34 & - \\
\hline
\end{tabular}

*Equilibrium facilitation factor $=30.0$.

t Numerical solution using orthogonal collocation on finite elements.

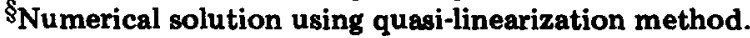


equilibrium facilitation. The fact that we were able to solve the equations even for such thick films represents a considerable extension in our capability to handle such systems. The effort involved does not increase substantially as the film thickness changes from $10 \mu \mathrm{m}$ ( 7 elements needed for the final convergent solutions) to $2.0 \mathrm{~cm}$ (26 elements needed for the final convergent solution). This is due to the ability of the present procedure to locate the elements where the solution is the most important. Table 2 illustrates how this was done for a membrane thickness of $2.0 \mathrm{~cm}$. The element locations were, of course, decided by examining the residuals at the element end points. The procedure does not require any prior knowledge of the boundary layer region. The boundary region is selectively scanned until enough elements are located in the boundary layer region to give very accurate results. Smith et al. [11] estimate (Fig. 5 of their paper) that the boundary layer thicknesses for this case are of the order of $1 \mu \mathrm{m}$. A procedure such as the one used by Kutchai et al. [15] where grid points were equally spaced would obviously require a large number of grid points to have any at all in the boundary layer region (about 20,000 for a membrane thickness of $2 \mathrm{~cm}$ ) where the solution is the most important. A comparison of our results with theirs indicates that although the difference is small ( $<2 \%$ in all cases), it does increase as the membrane thickness increases as expected.

Recently a new numerical procedure for solving these problems has been introduced by Nedelman and Rubinow (32). The procedure uses finite difference method with non-uniform grid points which allows the grid points to be concentrated in the boundary region. The procedure, however, does not incorporate any systematic way to adjust the location of grid points whereas in the finite element method the element locations can be systematically adjusted to get fast and accurate results. In the method of Nedelman and Rubinow, the two differential equations were combined in one by introducing two unknown parameters. These parameters have to be guessed at each stage of solution, resulting non-linear equations have to be solved iteratively and the new guesses for the parameters have to be found. The procedure is con-

\section{TABLE 2}

Typical sequence of element locations for $\mathrm{CO}$ facilitation $(L=2.0 \mathrm{~cm})$

\begin{tabular}{ll}
\hline $\begin{array}{l}\text { Pass through } \\
\text { the program }\end{array}$ & Element locations \\
\hline 1 & $0 ., 1.0$ \\
2 & $0 ., .1, .9,1.0$ \\
3 & $0 ., .01, .05, .1, .5, .9, .95, .99,1.0$ \\
4 & $0 ., .001, .005, .01, .05, .1, .3, .5, .7, .9, .95, .99, .995, .999,1.0$ \\
5 & $0 ., .0001, .0005, .001, .005, .01, .05, .1, .2, .3, .4, .5, .6, .7, .9, .95, .99$, \\
& $.995, .999, .9995, .9999,1.0$ \\
6 & $0 ., .0001, .0005, .001, .005, .01, .03, .05, .075, .1, .15, .2, .25, .3$, \\
& $.4, .5, .6, .7, .8, .9, .95, .99, .995, .999, .9995, .9997, .9999,1.0$ \\
\hline
\end{tabular}


tinued until the convergence is obtained. This procedure requires many iterations and a large computational effort. In contrast, finite elements procedure requires iterative solution of non-linear equations just once. Both the quasilinearization methods of Kuchai et al. and finite difference methods of Nedelman and Rubinow require good starting guesses. The present finite element method is capable of generating solutions even with poor starting guesses fairly inexpensively if the procedure explained below is followed.

From Table 2 we see that the solution was started at fairly small number of elements. One must follow this scheme to save on the computational costs. The reason for starting with small number of elements is that even though the lower order solutions are not very accurate (as is discussed later), they are important in guiding the solution in the right direction with considerable saving in computational effort. A starting solution such as $\bar{C}_{\mathrm{B}}=C_{\mathrm{T}} / C_{\mathrm{A}}^{0}$ and $\bar{C}_{\mathrm{A}}=1.0$, throughout the membrane for a small number of elements would converge for a fairly low computational cost. The converged solution would be a good guess for the next higher number of elements and would consequently reduce the number of iterations required for the higher order solutions where each iteration is more expensive due to larger system size. If a starting solution such as the one just mentioned was used for a large number of elements it would require large number of iterations (at a considerable computational cost) and may not converge at all. Each of the solutions listed in Table 1 required less than 2.0 sec in CPU time and the NewtonRaphson procedure converged in 3-5 iterations in each case, following the procedure just outlined.

The procedure described above can also be used to study the effect of various parameters on the solution. To study the effect of various parameters one uses the final converged solution for one set of parameters as the starting solution for the new set of parameters. The solution thus obtained would then be the solution for the new set of parameters. This procedure would be satisfactory if changing the parameters does not alter the solution substantially, otherwise the improvements in the solution would have to be made using the residual criterion.

Figure 3 illustrates how the error in the solution obtained by our method decreases as the number of elements increase. The results are plotted with membrane thickness as a parameter. The results indicate that initially the error does not decrease as rapidly but as additional elements are placed in appropriate locations, the error in the solution decreases very rapidly. This behaviour is obviously a desirable one. It also lends confidence to the lower order solutions. One can also observe another trend from Fig. 3. As the membrane thickness is increased, higher and higher order solutions are needed to obtain results of similar accuracy. This is not unexpected because as the membrane thickness increases, boundary layer thickness becomes proportionately smaller and the problem becomes more difficult to solve.

The approximate solutions of Smith et al. [11] and Kruezer and Hoofd [29] match well the results of Kutchai et al. [15], which are fairly close to 


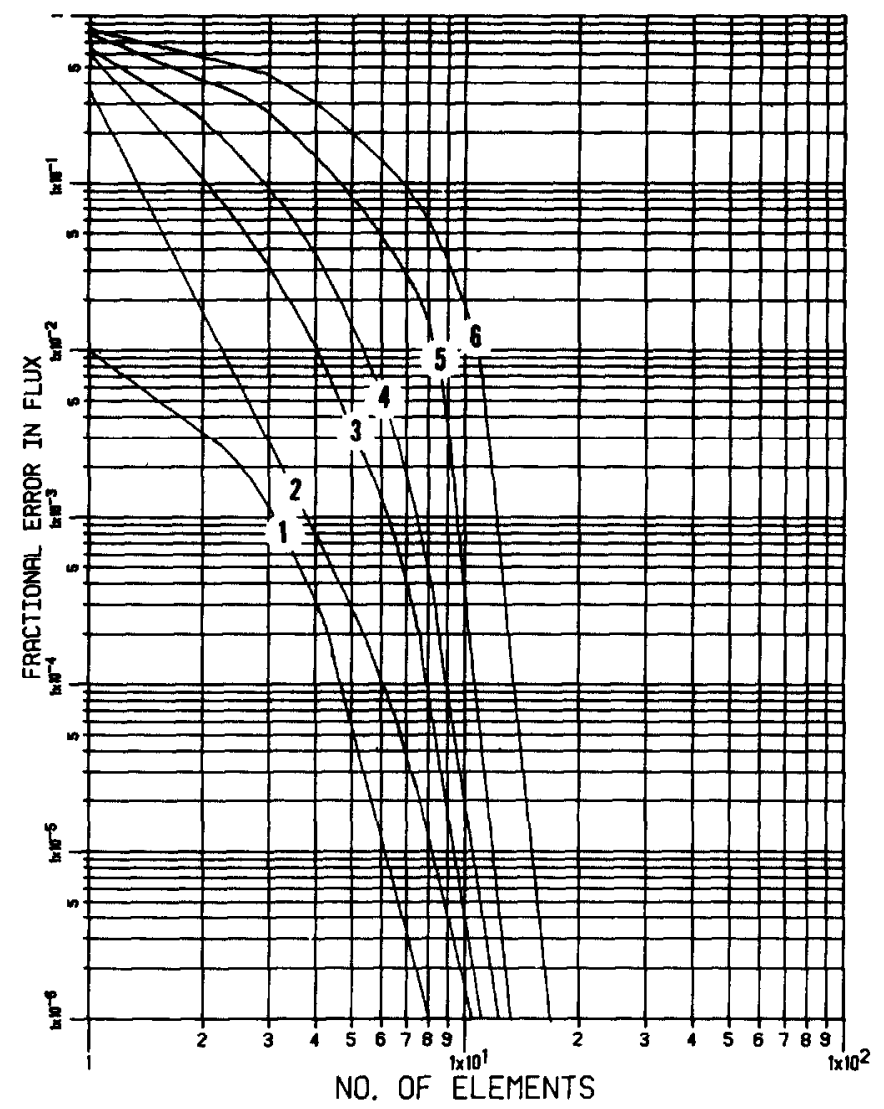

Fig. 3. Fractional error in flux as a function of number of elements for $\mathrm{CO}$ facilitation $(1, L=10 \mu \mathrm{m} ; 2, L=50 \mu \mathrm{m} ; 3, L=100 \mu \mathrm{m} ; 4, L=150 \mu \mathrm{m} ; 5, L=300 \mu \mathrm{m} ; 6, L=500 \mu \mathrm{m})$.

our results (Table 1). However, as Kutchai et al. [15] pointed out that they were not able to explain the experimental results of Mochizuki \& Forster [22] with the set of physical constants used by them. Kruezer and Hoofd [29] tried to explain the results of Mochizuki and Forster [22] using a new set of physical constants. Table 3 compares the results obtained using their approximate method and our exact numerical solution for the experimental conditions of Mochizuki and Forster. The results of the Kruezer-Hoofd's approximate solution would appear to have a satisfactory agreement with the experimental results. But comparison with the exact numerical solution indicates that the results of their calculations are in substantial error (up to $70 \%$ ).

Results of these calculations point out that an approximate method which works in one situation (compare results of Kruezer-Hoofd method with the numerical results of Kutchai et al.) may become fairly inaccurate in a seemingly similar situation. They also point out that even though an approx- 
TABLE 3

Summary of results on CO facilitation (Physical constants from Kruezer \& Hoofd [29])

\begin{tabular}{llccl}
\hline $\begin{array}{l}P \\
\text { (\% atm) }\end{array}$ & $F^{*}$ & $F^{* *}$ & $F^{* * *}$ & $\begin{array}{l}\text { \% Error in } \\
\text { Kruezer-Hoofd } \\
\text { method }^{\dagger}\end{array}$ \\
\hline 0.065 & 23.3 & 28.4 & 22.5 & 26.2 \\
0.27 & 5.00 & 6.00 & 5.06 & 18.6 \\
0.58 & 2.91 & 3.00 & 2.03 & 47.8 \\
1.67 & 0.70 & 0.70 & 0.41 & 70.7 \\
\hline
\end{tabular}

*Experimental results of Mochizuki and Forster [22], Fig. 2.

**Approximate method of Kruezer and Hoofd [29], Fig. 3.

***Numerical results using orthogonal collocation on finite elements.

${ }^{+}$The error in Kruezer-Hoofd's method was calculated by comparing their results with the results using orthogonal collocation. The comparison with the experimental results was not made because in actual experiments downstream concentration of $\mathrm{CO}$ was not zero, while both Kruezer-Hoofd's method and the present numerical procedure use this value in the calculations.

imate solution may agree with the experimental results, it is not a sufficient condition for its validity. Why does the Kruezer -Hoofd method fail in this case while comparing favorably with the numerical results of Kutchai et al. for a similar case? We believe the explanation lies in the fact that the value of the forward rate constant used by Kruezer and Hoofd in this case is two orders of magnitude higher than the value used in the calculations of Kutchai et al. [15] with other parameters remaining nearly the same. This pushes the system towards the so called "near diffusion or low facilitation regime" where the Kruezer and Hoofd [29] method is no longer accurate. This explanation is also supported by the results in Table 3 where error in Kruezer-Hoofd method is seen to increase as the facilitation factor decreases or the upstream CO partial pressure increases. We expect that as upstream CO partial pressure is increased further, the Kruezer-Hoofd method will become much worse because as we will see later the facilitation factor decreases very rapidly as upstream CO partial pressure is increased.

This example obviously points out the importance of numerical methods.

Unlike Kruezer-Hoofd's [29] method some approximate methods have their own criterion of validity $[8,11,32]$, but these criteria are so bruad that except in extreme cases one does not know how good an approximate solution is going to be, and one has to use the numerical methods to obtain reliable solutions.

We next discuss the effect of high upstream partial pressures on facilitated CO transport using the parameters of Kruezer and Hoofd [29]. The results are shown in Fig. 4. We find that the facilitation factor decreases very rapidly as the upstream CO partial pressure increases. Similar results are reported by Nedelman and Rubinow [32] for a different set of parameters. This should 


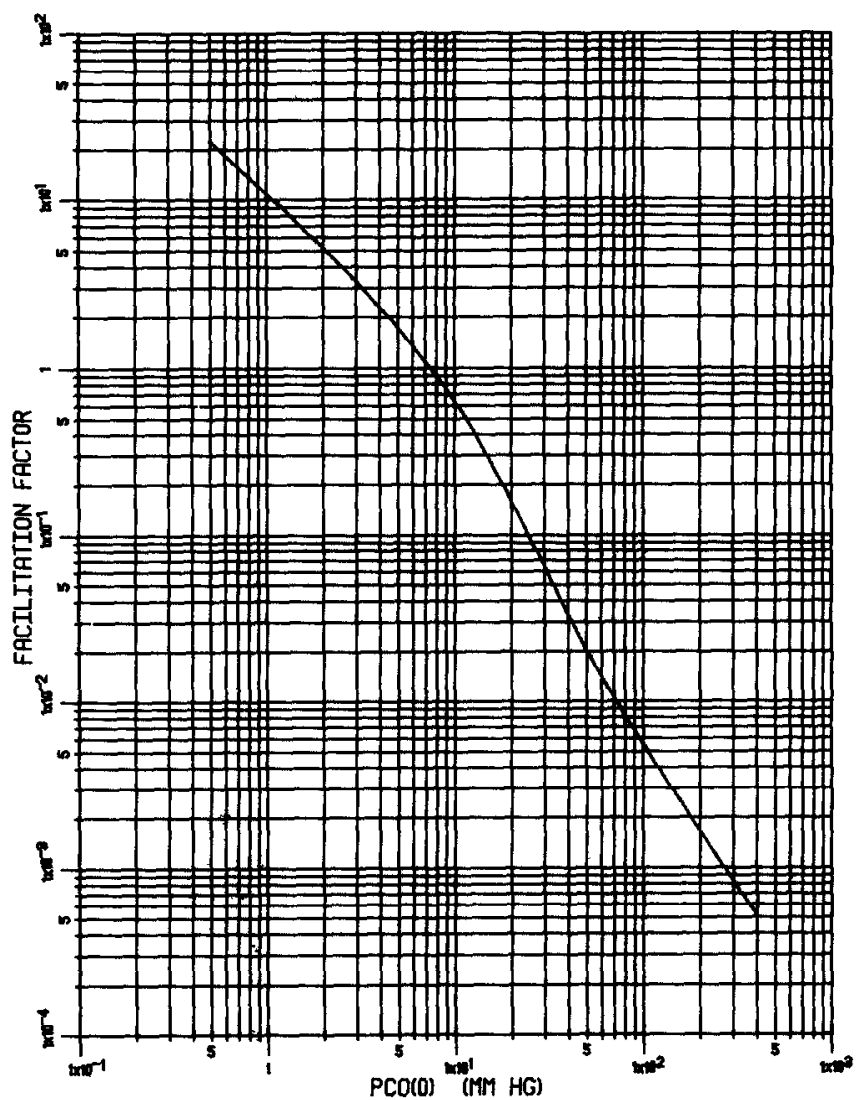

Fig. 4. Effect of upstream CO partial pressure on facilitation factor (Physical constants of Kruezer and Hoofd [29]).

account for no facilitations observed by Wittenberg [28] in his experiments.

We have also solved the equations for facilitated transport of oxygen by hemoglobin. The equations are identical to the ones for carbon monoxide. The physical constants of Kutchai et al. [15] were used for this case also. Comparisons of our results with Kutchai et al.'s results again indicated that as membrane thickness was increased the difference between the two results increased. A plot of fractional error in flux as a function of number of elements with layer thickness as a parameter had characteristics very similar to the same plot for CO (Fig. 3).

\section{The facilitation of carbon dioxide by bicarbonate solutions}

This example is included here to illustrate the use of the numerical technique for a system with complex kinetics. The equations solved correspond to uncatalyzed kinetic scheme for facilitated $\mathrm{CO}_{2}$ transport as given by Suchdeo and Schultz [31]. Equation (26) of their paper can be re-written 
as eqns. (36) and (37) below:

$$
\begin{aligned}
& \frac{\mathrm{d}^{2} \bar{C}_{\mathrm{A}}}{\mathrm{d} \bar{x}^{2}}=P\left(\bar{C}_{\mathrm{A}}-Q \frac{\bar{C}_{\mathrm{B}}^{2}}{1-\bar{C}_{\mathrm{B}}}\right)\left(1+R \frac{1-\bar{C}_{\mathrm{B}}}{\bar{C}_{\mathrm{B}}}\right) \\
& \frac{\mathrm{d}^{2} \bar{C}_{\mathrm{B}}}{\mathrm{d} \bar{x}^{2}}=S\left(\bar{C}_{\mathrm{A}}-Q \frac{\bar{C}_{\mathrm{B}}^{2}}{1-\bar{C}_{\mathrm{B}}}\right)\left(1+R \frac{1-\bar{C}_{\mathrm{B}}}{\bar{C}_{\mathrm{B}}}\right)
\end{aligned}
$$

where $\bar{C}_{\mathrm{A}}=C_{\mathrm{A}} / C_{\mathrm{A}}{ }^{0}$ and $\bar{C}_{\mathrm{B}}=C_{\mathrm{B}} / C_{\mathrm{T}}$.

The boundary conditions can be written as

$$
\begin{array}{ll}
\bar{C}_{\mathrm{A}}=1.0, \quad \frac{\mathrm{d} \bar{C}_{\mathrm{B}}}{\mathrm{d} \bar{x}}=0 & \text { at } \bar{x}=0 \\
\bar{C}_{\mathrm{A}}=C_{\mathrm{A}}^{\mathrm{L}} / C_{\mathrm{A}}{ }^{0}, \frac{\mathrm{d} \bar{C}_{\mathrm{B}}}{\mathrm{d} \bar{x}}=0 & \text { at } \bar{x}=1
\end{array}
$$

The correspondences between various quantities in these equations and the ones in the original paper (using the nomenclature used therein) are given below:

$\bar{C}_{\mathrm{A}}=\widetilde{C}_{1}=\hat{C}_{1} / \hat{C}_{1}{ }^{\circ}$

$\bar{C}_{\mathrm{B}}=C_{3}=\hat{C}_{3} / \hat{C}_{6}^{0}$

$C_{\mathrm{A}}{ }^{0}=\hat{C}_{1}^{0}$ and $C_{\mathrm{A}}^{L}=\rho \hat{C}_{1}^{0}$

$C_{\mathrm{T}}=\hat{C}_{6}{ }^{0}$

$P=\alpha_{1}^{2}=k_{1} L^{2} / D_{\mathrm{A}}$

$Q=M=2 \hat{C}_{6}^{0} /\left(K H_{1}\right) P_{0}$

$R=n=k_{2} k_{6} / 2 k_{1} k_{4}$

and

$S=-\alpha_{1}{ }^{2} / g$, where $g=D_{3} \hat{C}_{6}{ }^{0} / 2\left(D_{1} H_{1}\right) P_{0}$

These equations were solved for conditions corresponding to Fig. 1 of Suchdeo and Schultz [31]. The routines needed for this case are included in the users' manual. Facilitation factor values for different diffusion reaction resistances, $\alpha_{1}$ 's, are plotted in Fig. 5. The numerical values of the results are included in Table 4. This table indicates that the computational effort is small even for very thick membranes. It can also be seen that equilibrium facilitation has not been achieved even for a membrane thickness of $20 \mathrm{~cm}$. Figure 6 shows the concentration profiles as a function of number of elements for a layer thickness of $1 \mathrm{~cm}$. It is clear that as the number of elements are increased the concentration profiles converge very rapidly again indicating that the lower order solutions are also fairly accurate. The fractional error in the flux 


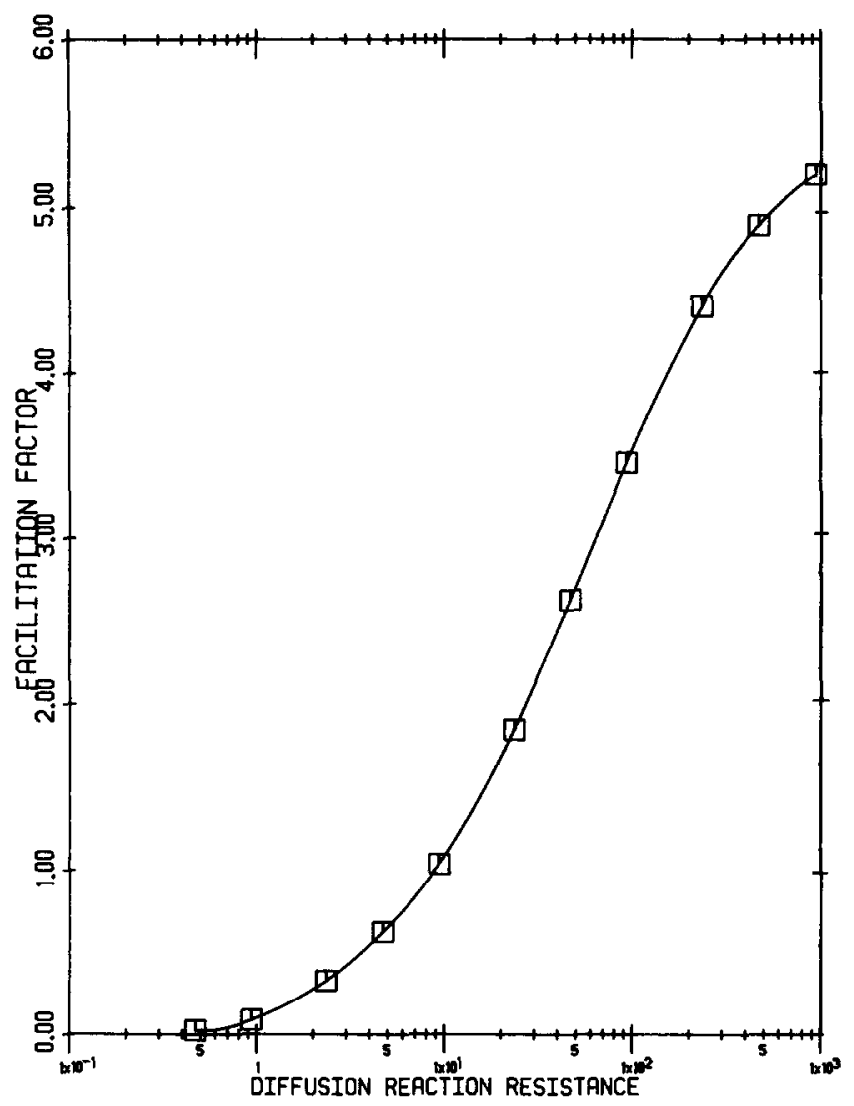

Fig. 5. Facilitation factor, $F$, against diffusion-reaction resistance $\alpha_{1}$, curve for $\mathrm{CO}_{2}$ facilitation. $\square$, points where the computations were made.

\section{TABLE 4}

The dependence of the $\mathrm{CO}_{2}$ facilitation* on layer thickness $\left(P_{\mathrm{CO}_{2}}(0)=30 \mathrm{~mm} \mathrm{Hg}\right.$ and $\left.P_{\mathrm{CO}_{2}}(L)=3 \mathrm{~mm} \mathrm{Hg}\right)$

\begin{tabular}{lrrlll}
\hline $\begin{array}{l}L \\
(\mathrm{~cm})\end{array}$ & \multicolumn{1}{l}{$\alpha_{1}$} & $M$ & $C_{\mathrm{B}}(0) / C_{\mathrm{T}}$ & $C_{\mathrm{B}}(L) / C_{\mathrm{T}}$ & $F$ \\
\hline $\mathbf{0 . 0 1}$ & $\mathbf{0 . 4 7}$ & 4 & 0.9659 & 0.9638 & 0.0249 \\
0.02 & 0.94 & 7 & 0.9681 & 0.9606 & 0.0885 \\
0.05 & 2.35 & 9 & 0.9747 & 0.9468 & 0.326 \\
0.1 & 4.70 & 10 & 0.9784 & 0.9255 & 0.622 \\
0.2 & 9.40 & 12 & 0.9799 & 0.8916 & 1.04 \\
0.5 & 23.5 & 13 & 0.9808 & 0.8236 & 1.85 \\
1.0 & 47.0 & 15 & 0.9812 & 0.7575 & 2.63 \\
2.0 & 94.0 & 17 & 0.9814 & 0.6873 & 3.46 \\
5.0 & 235.0 & 19 & 0.9815 & 0.6070 & 4.40 \\
10.0 & 470.0 & 19 & 0.9816 & 0.5652 & 4.89 \\
20.0 & 940.0 & 22 & 0.9817 & 0.5391 & 5.20 \\
\hline
\end{tabular}

*Equilibrium facilitation factor $=5.58$. 


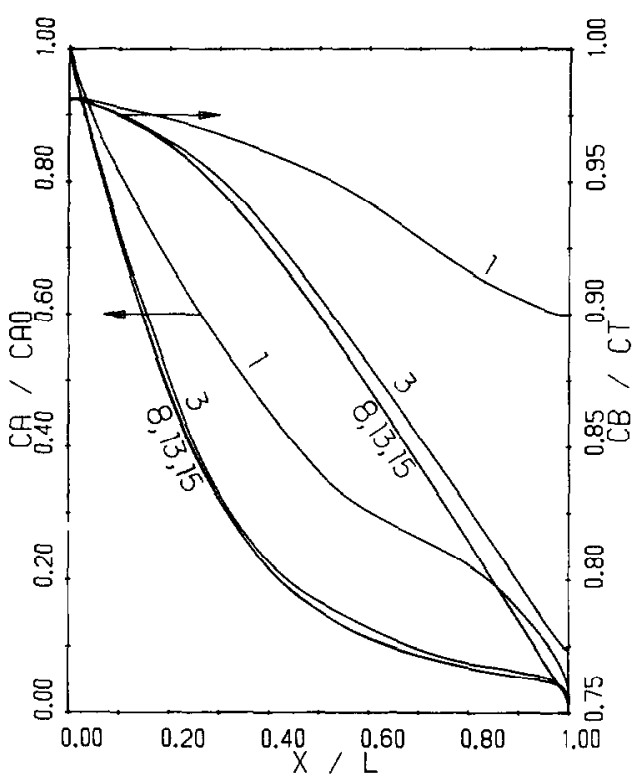

Fig. 6. Concentration profiles for $\mathrm{CO}_{2}$ facilitation as a function of number of elements for layer thickness of $1.0 \mathrm{~cm}$.

in all cases was found to decrease very rapidly as the number of elements were increased. This trend is very similar to the case of $\mathrm{CO}$ facilitation. Suchdeo and Schultz reported that at high values of $\alpha_{1}$ (values greater than 20) convergence could not be obtained with their quasi-linearization procedure.

However as Table 4 indicates that we were able to obtain solutions for much higher values of $\alpha_{1}$ without much difficulty. This again indicates the superiority of the present method. The results obtained using two methods, for the range they are available, are comparable in accuracy.

\section{The facilitation of NO through ferrous chloride solutions}

This case was first solved using the values of the physical constants used by Ward [17], who computed the results for various carrier concentrations. The results indicated that a fairly small number of elements $(<8)$ were needed to solve equations for this case. Good agreement was found between numerical and experimental results. A plot of fractional error in flux vs. number of elements with carrier concentration as a parameter showed no obvious dependence of carrier concentration on the error in flux.

We next solved the equations for this system using a hypothetical set of physical constants. These constants were same as used by Ward [17] except for the diffusivity of $\mathrm{FeNO}^{2+}$. Instead of assuming $D_{\mathrm{Fe}^{2+}}=D_{\mathrm{FeNO}^{2+}}=2 \times 10^{-6}$ $\mathrm{cm}^{2} / \mathrm{sec}$, we used $D_{\mathrm{Fe}^{2+}}=2 \times 10^{-6} \mathrm{~cm}^{2} / \mathrm{sec}$ and $D_{\mathrm{FeNO}^{2+}}=1 \times 10^{-6} \mathrm{~cm}^{2} / \mathrm{sec}$. This case then falls under the category in which the diffusivities of the carrier and its complex are not same. The de-dimensionalized equations for this case 
are written below:

$\frac{\mathrm{d}^{2} \bar{C}_{\mathrm{A}}}{\mathrm{d} \bar{x}^{2}}=P\left(Q \bar{C}_{\mathrm{A}} \bar{C}_{\mathrm{B}}-T+R \bar{C}_{\mathrm{B}}\right)$

and

$\frac{\mathrm{d}^{2} \bar{C}_{\mathrm{B}}}{\mathrm{d} \bar{x}^{2}}=S\left(Q \bar{C}_{\mathrm{A}} \bar{C}_{\mathrm{B}}-T+R \bar{C}_{\mathrm{B}}\right)$

where

$P=k_{2} L^{2} / D_{\mathrm{A}}, \quad S=k_{2} L^{2} / D_{\mathrm{B}}, \quad Q=\left(k_{1} / k_{2}\right) C_{\mathrm{A}}^{0}$,

$R=D_{\mathrm{B}} / D_{\mathrm{AB}}$ and $T=C^{*} / D_{\mathrm{AB}} C_{\mathrm{A}}{ }^{0}$

The boundary conditions are given by eqn. (14).

The procedure to solve for the concentration profiles and the flux for this case involves obtaining a guess value of $C^{*}$ from eqn. (6) by substituting $D_{\mathrm{B}}=D_{\mathrm{AB}}=\left(D_{\mathrm{AB}}+D_{\mathrm{B}}\right) / 2$ and $C_{\mathrm{B}}+C_{\mathrm{AB}}=C_{\mathrm{T}}$. The computational procedure is then started for a small number of elements. Once this solution converges, another value for $C^{*}$ is obtained using eqn. (8). The value of $C^{*}$ to be used for next higher number of elements is found by comparing the calculated and assumed values of $C^{*}$. This procedure is continued until the residuals at element end points are fairly small. The convergence in the values of $C^{*}$ would probably be obtained by then. If that is not the case, one has to

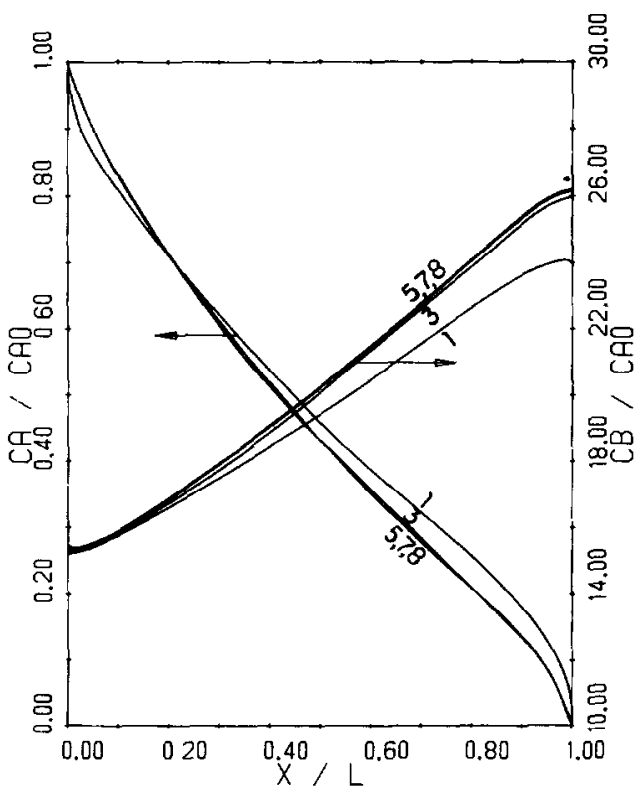

Fig. 7. Concentration profiles for NO facilitation as a function of number of elements for a carrier conc. of $0.1 M$ (unequal diffusivities case). 
iteratively vary the value of $C^{*}$ till the values converge. The equations for this system were solved for a carrier concentration of $0.1 M$. Use was made of routine DIFCOR to make corrections for the differences in the diffusivities. Figure 7 shows the concentration profiles for various passes through the computer program. It is clear from this figure that the concentration profiles in this case also converge very rapidly. The numerical values of the results for this case are included in Table 5. The results indicate that both the flux and the value of $C^{*}$ converge very rapidly. This example illustrates that the present computational procedure can handle the unequal diffusivities case as efficiently as the equal diffusivities case. None of the computational procedures in the literature have attempted the solution of carriermediated transport equations for the case of unequal diffusivities which is an important case for facilitation involving small molecules. The complete set of routines required for this case are also included in the users' manual.

TABLE 5

Summary of the results for NO facilitation for the unequal diffusivity case $\left(C_{\mathrm{T}}=0.1 M\right)$

\begin{tabular}{lllll}
\hline$M$ & $\begin{array}{l}C^{*} \times 10^{10} \\
\text { (assumed) } \\
\text { (moles/cm sec) }\end{array}$ & $\begin{array}{l}C^{*} \times 10^{10} \\
\text { (calculated) } \\
\text { (moles/cm sec) }\end{array}$ & $\begin{array}{l}\text { Flux } \times 10^{10} \\
\left(\text { moles } / \mathrm{cm}^{2} \mathrm{sec}\right)\end{array}$ & $F$ \\
\hline 1 & 1.5 & 1.51262 & 8.004 & 1.098 \\
3 & 1.523 & 1.53127 & 9.277 & 1.431 \\
5 & 1.533 & 1.53486 & 9.320 & 1.443 \\
7 & 1.536 & 1.53593 & 9.332 & 1.446 \\
8 & 1.53591 & 1.53591 & 9.332 & 1.446 \\
\hline
\end{tabular}

\section{Conclusions}

The versatility of the method of orthogonal collocation in solving the boundary layer type of problems involving carrier-mediated transport systems has been demonstrated. Comparison with other numerical methods indicates that the method is more efficient and has very good convergence properties. The efficiency in storage results from the blockdiagonal nature of the equations and efficient LU decomposition of blockdiagonal matrices leads to the efficiency in computations. The residual criterion enables the placement of the elements in appropriate places leading to a very fast convergence. The interactive nature of the computer program for the method allows a very easy implementation of the residual criterion and the solutions can be easily obtained without any prior knowledge. Applications to three different physical systems (CO transport through hemoglobin solutions, $\mathrm{CO}_{2}$ transport through bicarbonate solutions, and NO transport through ferrous chloride solutions) shows that the calculations can be made for a wide variety of situations fairly easily. Unlike the semi- 
analytical solutions which are not always reliable, the present numerical method can be used with confidence for all situations. The computer program for the method can be used for systems with unequal diffusivities for carrier and its complex or for systems with different kinetics by making minor modifications and has a wider applicability in solving steady state problems involving diffusion and chemical reaction.

\section{Acknowledgement}

This work was supported by NSF Grant\#NSF/CPE 78-13316.

\section{Appendix}

The construction of various user supplied routines needed for the computer program, CARMED, is illustrated in this Appendix. Equation (12)-(14) of the text are used for this purpose. A listing of various user supplied routines is included in Fig. 8.

The routine PARAM calculates the dimensionless parameters of the system such as $P, Q, S$ and $T$ in eqns. (12) and (13). The values of the physical constants are passed to this routine via a COMMON block PCOM. This routine also calculates premultipliers, $Z_{1}$ and $Z_{2}$, which when multiplied by $\left(\bar{C}_{\mathrm{A}}^{0}-\bar{C}_{\mathrm{A}}{ }^{1}\right)$ and $\left(\bar{C}_{\mathrm{B}}^{1}-\bar{C}_{\mathrm{B}}^{0}\right)$ would give unfacilitated and facilitated fluxes respectively. COMMON block QCOM in this routine contains the dimensionless parameters.

The routine FUNC is used to define the kinetic expressions (right hand side of eqns. (12) and (13)). It uses the dimensionless parameters calculated by routine PARAM which are passed to it via COMMON block QCOM.

The routine PDERV is used to evaluate the partial derivatives of the kinetic functions $\mathrm{FX}(1)$ and $\mathrm{FX}(2)$ with respect to the concentrations $\mathrm{CA}$ and $\mathrm{CB}$. We have $\mathrm{DX}(1, \mathrm{I})=\partial \mathrm{FX}(\mathrm{I}) / \partial \mathrm{CA}$ and $\mathrm{DX}(2, \mathrm{I})=\partial \mathrm{FX}(\mathrm{I}) / \partial \mathrm{CB}$.

The routine BNDRY is used to define the boundary concentrations of CA. We have CABND(1) $=C_{\mathrm{A}}{ }^{0} / C_{\mathrm{A}}{ }^{0}$ and $\mathrm{CABND}(2)=C_{\mathrm{A}}{ }^{0} / C_{\mathrm{A}}{ }^{L}$.

The routine DIFCOR is used to calculate the integral in eqn. (8) of the text when $D_{\mathrm{B}} \neq D_{\mathrm{AB}}$. The complete version of this routine for example 3 is included in the users' manual. However, since for the present case $D_{\mathrm{B}}=D_{\mathrm{AB}}$, a dummy routine is substituted to satisfy the loader.

When a problem different than the one discussed here is to be solved, one has to rewrite routines PARAM, FUNC and PDERV as explained above. COMMON block PCOM has to be redefined in routine BNDRY and COMMON blocks PCOM and QCOM have to be re-defined in the MAIN program. Besides these the READ statements in the MAIN program have to be changed to read all the physical constants of the system.

This example illustrates how easily the computer program can be used for different systems. 
THIS SUBROUTIIIE CALCULATES THE PARAYTTERS OF THE DIFFEREMTIAL

EOUAT IOUS USING THE PMTSICAL COUSTATS SUPPLIED OY THE MAIN

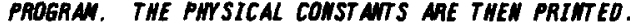

INP LICIT REAL*B(A-H,O-Z)

LOGICAL COEFF

COMONIPCONIAK1 , AK2, AL, CA, CAD, CAL, CST AR, CT, DA,DAB,DB

COMOHIOCONIP,O,S,T,21,22

$P=A K 2 * A L * A L I D A$

$Q=A K T / A K 2 * C A$

$T=C T / C A$

$5=A K 2 * A L * A L I D B$

IMITE $16,200 / A K 1, A K 2, A L, O A, D B, C T, C A, C A L$

COEFF $=$.FALSE.

$c$
$c$

- . CALCULATE THE PREMULTIPLIEAS FOR THE FLUX EXPRESSIONS

21 FOR (CAO-CAL) ANO 22 FOR (CBL-CBO)...

$21=D A * C A / A L$

$Z 2=D B * C A / A L$

C

FORMAT FOR THE OUTPUT ST ATENENT

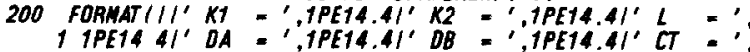

1 IPE14 $4 I^{\prime} C A=$, IPE14.4I' CAL $=$ ', IPE14.4I'

RETURN

END

SUBROUTIHE FUNCICA, CB, FX)

THIS ROUT IHE CALCULATES THE KINETIC EXPRESSIONS FOR COMPONENTS

$A$ AND $B$

INPLICIT REAL*8(A-H, O-Z)

CONMON IOCOMIP, $0,5,7,21,22$

DIMENSION $F X(2)$

$F X(1)=P *(0 * C A * C B-T+C B)$

$F X(2)=S *(0 * C A * C B-T+C B)$

RETURN

END

SUBROUTINE POERV (CA, CB, DX)

c THIS ROUTINE CALCULATES THE PART IAL DERIVATIVES OF THE KIMETIC EXPRESSIONS FX(1) ANO FX(2) IN ROUT INE FUNC

INP LICIT REAL*8(A-H,O-2)

COMNNIOCONIP,O, S, T, 21,22

OINENSION DX 2,21

$0 \times(1,1)=P * 0 * C B$

$D X(1,2)=P *(0 * C A+1.00)$

$B \times(2,1)=S * 0 * C B$

$D \times(2,2)=S *(0 * C A+100)$

RETURN

END

C

SUBROUTINE BNORY (CABNO)

C THIS ROUTIHE RETURHS THE VALUE OF CA $A T X-0 \& 1$.

C

INPLICIT REAL*8(A-H,0-Z)

CONHONIPCONIAK1 AK2 , AL , CA, CAO , CAL, CST AR, CT , OA, OAB, OB

DIMENSION CABND $(2)$

$C A B N D(1)=C A D$

$C A B M D(2)=C A L$

RETURN

END

SUBROUT INE DIFCOR(CST ARC,DELX, NBLOCK, HOIAB, NDINS, XOLO,W)

$c$
$c$
$c$
$c$

THIS ROUTIME IS USED TO MAKE CORAECTIONS FOR UMEQUAL

OIFFUSIVITIES CASE

RETURN

Fig. 8. Listing of routines PARAM, FUNC, PDERV, BNDRY and DIFCOR for CO facilitation. 


\section{List of symbols}

$a, b \quad$ Coefficients of the series (eqn. (18)), also the boundary conditions (eqn. (29)).

$a_{i}=\quad$ Coefficients of the series in eqn. (18).

$\bar{A}, \overline{\bar{B}} \quad$ Square matrices dependent on the roots of the orthogonal polynomials (eqns. (24) and (25)).

C* An integration constant.

$C_{i} \quad$ Concentration of the species $i\left(\operatorname{moles} / \mathrm{cm}^{3}\right)$.

$\bar{C}_{i} \quad$ Dimensionless concentration of species $i$.

$C_{\mathrm{T}} \quad$ Total carrier concentration $\left(\mathrm{moles} / \mathrm{cm}^{3}\right)$.

$C_{i}{ }^{0}, C_{i}^{L} \quad$ Concentrations of species $i$ at $x=0$ and $L\left(\right.$ moles $\left./ \mathrm{cm}^{3}\right)$.

$\bar{C}_{i}^{0}, \bar{C}_{t}^{1} \quad$ Dimensionless concentrations of species $i$ at $\bar{x}=0$ and 1 .

$C_{\mathrm{A}_{i}}^{l}, C_{\mathrm{B}_{2}}{ }^{2}$ Values of $C_{\mathrm{A}}$ and $C_{\mathrm{B}}$ at the $i$ th collocation point in the $l$ th element.

$C_{i}^{l}(x) \quad$ A function denoting the value of $C_{i}$ in $l$ th element.

$\bar{d} \quad$ A vector defined by eqn. (23).

$d_{i} \quad$ Constants in eqns. (19) and (20).

$d_{l}^{l} \quad$ Coefficients of the interpolating polynomial in $l$ th element (eqn. (35)).

$\underline{D}_{i} \quad$ Diffusion coefficient of species $i\left(\mathrm{~cm}^{2} / \mathrm{sec}\right)$.

$\overline{\bar{D}} \quad$ Square matrix defined by eqn. (22).

$\overline{\bar{E}} \quad$ Square matrix defined by eqn. (22).

$f(x, y) \quad$ A function of $x$ and $y$ (eqn. (17)).

$F \quad$ Facilitation factor.

$\bar{F}(\bar{y}) \quad$ A vector representing the blockdiagonal system of equations.

$\overline{\bar{F}} \quad$ Square matrix defined by eqn. (22).

$\bar{G} \quad$ Vector defined by eqn. (27).

$\overline{\bar{J}}$

Jacobian matrix for the blockdiagonal system of equations.

$k_{1} \quad$ Forward rate constant $\left(\mathrm{cm}^{3} /\right.$ moles sec $)$.

$k_{2} \quad$ Reverse rate constant $\left(\mathrm{sec}^{-1}\right)$.

$L \quad$ Membrane thickness (cm).

$M \quad$ Number of elements.

$N \quad$ Number of interior collocation points in each element.

$N_{\mathrm{A}} \quad$ Flux of the permeant A (moles $\left./ \mathrm{cm}^{2} \mathrm{sec}\right)$.

$P \quad$ Damköhler number (eqn. (13)).

$P_{l} \quad$ Partial pressure of the component $i(\mathrm{mmHg})$.

$P_{l}(x) \quad$ Orthogonal polynomial of $i$ th order.

$Q \quad$ A dimensionless parameter (eqn. (13)).

$r_{\mathrm{l}} \quad$ Reaction rate for species $i\left(\mathrm{moles} / \mathrm{cm}^{3} \mathrm{sec}\right)$.

$S \quad$ Damköhler number (eqn. (13)).

$T \quad$ A dimensionless parameter (eqn. (13)).

$u \quad$ A transformed distance coordinate.

$\bar{W} \quad$ Vector defined by eqn. (27).

$x \quad$ A distance coordinate.

$\bar{x} \quad$ Dimensionless distance coordinate. 
$\Delta X_{l} \quad$ Length of the lth element.

$\bar{y} \quad$ The solution vector.

$\bar{y}^{k} \quad$ The solution vector after $k$ th iteration in the Newton-Raphson procedure.

\section{References}

1 P.F. Scholander, Oxygen transport through hemoglobin solutions, Science, 131 (1960) 585.

2 J.S. Schultz, J.D. Goddard and S.R. Suchdeo, Facilitated transport via carrier-mediated diffusion in membranes, Part I, Mechanistic aspects, experimental systems and characteristic regimes, AIChE J., 20 (1974) 417.

3 J.S. Schultz, Carrier-mediated transport in liquid-liquid membrane systems, in: N.N. Li (Ed.), Recent Developments in Separation Science, Vol. 3, CRC Press, Cleveland, Ohio, 1977.

4 D.R. Smith, R.J. Lander and J.A. Quinn, Carrier-mediated transport in synthetic membranes, in: N.N. Li (Ed.), Recent Developments in Separation Science, Vol. 3, CRC Press, Cleveland, Ohio (1977).

5 S.L. Matson, C.S. Herrick and W.J. Ward, Progress on the selective removal of $\mathrm{H}_{2} \mathrm{~S}$ from gasified coal using an immobilized liquid membrane, Ind. Eng. Chem. Process. Des. Develop., 16 (1977) 370.

6 J.D. Goddard, Further applications of carrier-mediated transport theory - A survey, Chem. Eng. Sci., 32 (1977) 795.

7 R.D. Hughes, E.F. Steigelmann and J.A. Mahoney, Olefin separation by facilitated transport membranes, Paper No. 1d, presented at AIChE 1981 spring national meeting, Houston, Texas.

8 J.D. Goddard, J.S. Schultz and R.J. Bassett, On membrane diffusion with nearequilibrium reaction, Chem. Eng. Sci., 25 (1970) 665.

9 F. Kruezer and L.J.C. Hoofd, Facilitated diffusion of oxygen in the presence of hemoglobin, Resp. Physiol., 8 (1970) 380.

10 F. Kruezer and L.J.C. Hoofd, Factors influencing facilitated diffusion of oxygen in the presence of hemoglobin and myoglobin, Resp. Physiol., 15 (1972) 104.

11 K.A. Smith, J.K. Meldon and C.K. Colton, An analysis of carrier-facilitated transport, AIChE J., 19 (1973) 102.

12 S.K. Friedlander and K.H. Keller, Mass transfer in reacting systems near equilibrium; Use of the affinity function, Chem. Eng. Sci., 20 (1965) 121.

13 D.R. Smith and J.A. Quinn, The prediction of facilitation factors for reaction augmented membrane transport, AIChE J., 25 (1979) 197.

14 L. Hoofd and F. Kruezer, The mathematical treatment of steady state diffusion of reacting species, AIChE Symp. Ser., 77 (1981) 123.

15 H. Kutchai, J.A. Jacquez and F.J. Mather, Nonequilibrium facilitated oxygen transport in hemoglobin solutions, Biophys. J., 10 (1970) 38.

16 S.R. Suchdeo and J.S. Schultz, Mass transfer of $\mathrm{CO}_{2}$ across membranes: Facilitation in the presence of bicarbonate ion and the enzyme carbonic anhydrase, Biochim. Biophys. Acta, 352 (1974) 412.

17 W.J. Ward, Analytical and experimental studies of facilitated transport, AIChE J., 16 (1970) 405.

18 G.F. Carey and B.A. Finlayson, Orthogonal collocation on finite elements, Chem. Eng. Sci., 30 (1975) 587.

19 J.S. Schultz, Carrier-mediated photodiffusion membranes, Science, 197 (1977) 1177.

20 J.S. Schultz, Facilitation of $\mathrm{CO}_{2}$ through layers with a spatial distribution of carbonic anhydrase, in: C. Bauer, G. Gros and H. Bartels (Eds.), Biophysics and Physiology of Carbon Dioxide, Springer-Verlag (Berlin), 1980. 
21 T.L. Donaldson and J.A. Quinn, Carbon dioxide transport through enzymatically active synthetic membranes, Chem. Eng. Sci., 30 (1975) 103.

22 M. Mochizuki and R.E. Forster, Diffusion of carbon monoxide through thin layers of hemoglobin solution, Science, 138 (1962) 897.

23 H.J. van Otiwerkerk, Facilitated diffusion in a tissue cylinder with an anoxic region, Pflügers Arch., Berlin, 372 (1977) 221.

24 J. Villadsen, Selected approximation methods for chemical engineering problems, Inst. for Kemiteknik Numer. Inst. Danmarks Tekniske Hфjskole, 1970.

25 B.A. Finlayson, The Method of Weighted Residuals and Variational Principles, Academic Press, New York, 1972.

26 B. Carnahan, H.A. Luther and J.O. Wilkes, Applied Numerical Methods, John Wiley \& Sons, New York, 1969.

27 G. Forsythe and C.B. Moler, Computer Solution of Linear Algebraic Systems, PrenticeHall, New Jersey, 1967.

28 J.B. Wittenberg, The molecular mechanism of hemoglobin facilitated oxygen diffusion, J. Biol. Chem., 241 (1966) 104.

29 F. Kruezer and L.J.C. Hoofd, Facilitated diffusion of $\mathrm{CO}$ and oxygen in the presence of hemoglobin or myoglobin, in J. Grote, D. Reneau and G. Thews (Eds.), Oxygen Transport to Tissue - II, Adv. Exper. Med. Biol., Plenum Press, New York and London, 75 (1976) 207.

30 J.D. Murray and J. Wyman, Facilitated diffusion, The case of carbon monoxide, Biol. Chem., 246 (1971) 5903.

31 S.R. Suchdeo and J.S. Schultz, The permeability of gases through reacting solutions: The carbon dioxide-bicarbonate membrane system, Chem. Eng. Sci., 29 (1974) 13.

32 J. Nedelman and S.I. Rubinow, Facilitation diffusion of oxygen and carbon monoxide in the large affinity regime, J. Math. Biol., 12 (1981) 73.

33 B.A. Finlayson, Nonlinear Analysis in Chemical Engineering, McGraw Hill, 1980. 